\title{
Bodenfeuchtedaten aus Fernerkundung für hydrologische Anwendungen
}

Zusammenfassung: Die Bodenfeuchtigkeit spielt eine zentrale Rolle im hydrologischen Kreislauf auf den verschiedensten Maßstabsskalen. Fernerkundungsmethoden, wie etwa die Aufnahme mit Mikrowellen, erlauben eine flächendeckende Beschreibung der Bodenfeuchte an der Landoberfläche. Diese kann als Input für hydrologische Modelle und für Wettervorhersagemodelle, sowie als Information für den Katastrophenschutz dienen. Dieser Beitrag gibt eine Einführung in die aktuellen Entwicklungen von Bodenfeuchtigkeitsprodukten. Anhand von Fallstudien in Österreich wird der Nutzen für hydrologisch-wasserwirtschaftliche Anwendungen aufgezeigt.

Remotely sensed soil moisture data for hydrological applications

Summary: Soil moisture plays a major role in the hydrologic cycle at a range of scales. Remote sensing methods, such as microwave techniques, provide spatial information on the land surface which can be used as an input to hydrological models and weather prediction models, and as information for disaster management. This paper provides an introduction into recent developments of soil moisture products. The value of these products for hydrological and water resources applications is illustrated by case studies in Austria.

\section{Einleitung}

Obwohl die Bodenfeuchte nur einen geringen Anteil am weltweiten Frischwasservorrat repräsentiert, ist ihr Einfluss auf den globalen Wasserkreislauf von großer Bedeutung. Sie stellt eine wichtige Ressource für Pflanze und Mensch dar, und bildet die Grundlage für die landwirtschaftliche Produktion. Die Bodenfeuchte

Institut für Photogrammetrie und Fernerkundung, TU Wien

2 Institut für Wasserbau und Ingenieurhydrologie, TU Wien ist eine mitbestimmende Größe bei der Interaktion zwischen Atmosphäre und Boden, und hat somit auch direkten Einfluss auf das regionale Klima und Wettergeschehen. Des Weiteren spielt die Bodenfeuchte bei der Abflussentstehung in Einzugsgebieten eine wichtige Rolle, da sie häufig der maßgebende Faktor für die Entstehung von oberflächlichem und oberflächennahem Abfluss ist. Die Berücksichtigung des Bodenwassergehalts ist somit ein entscheidender Bestandteil in der Modellierung hydrologischer Prozesse für Bilanzierungsaufgaben, Bewirtschaftungspläne und Hochwasservorhersagen. Am häufigsten kommen in diesem Zusammenhang kontinuierliche NiederschlagAbflussmodelle zum Einsatz, die die Komponenten des Wasserhaushaltes eines Einzugsgebietes als Funktion der Bodenfeuchte behandeln. Dabei ist die simulierte Bodenfeuchte eine modellinterne Zustandsgröße.

Im Regelfall liegt das Hauptaugenmerk bei der hydrologischen Modellierung auf der möglichst wirklichkeitsnahen Wiedergabe der Abflussverhältnisse in einem Einzugsgebiet. Dementsprechend erfolgt die Eichung der Modellparameter hauptsächlich anhand beobachteter Abflüsse. Diese Vorgangsweise bei der Parameterschätzung kann zu systematischen Fehlern der simulierten Bodenfeuchte führen, welche durch den Vergleich der simulierten mit den beobachten Abflussganglinien im Eichzeitraum nicht identifizierbar sind. Es liegt nahe, Bodenfeuchtemessungen in die Parameterschätzung einzubeziehen um systematische Fehler zu reduzieren und die Abflussprognose genauer zu simulieren.

Bei der Messung von Bodenfeuchte kann grundsätzlich zwischen zwei verschiedenen Methoden unterschieden werden; der Messung im Felde (direkt im Boden) und der Messung aus der Distanz mittels Fernerkundungsmethoden. Die beiden Methoden unterscheiden sich wesentlich durch die räumlichen und zeitlichen Skalen. Bei den Messungen vor Ort (in-situ) kann die zeitliche Dynamik der Bodenfeuchte in unterschiedlichen Bodentiefen kontinuierlich erfasst werden. Allerdings ist die Aussagekraft der Messer-

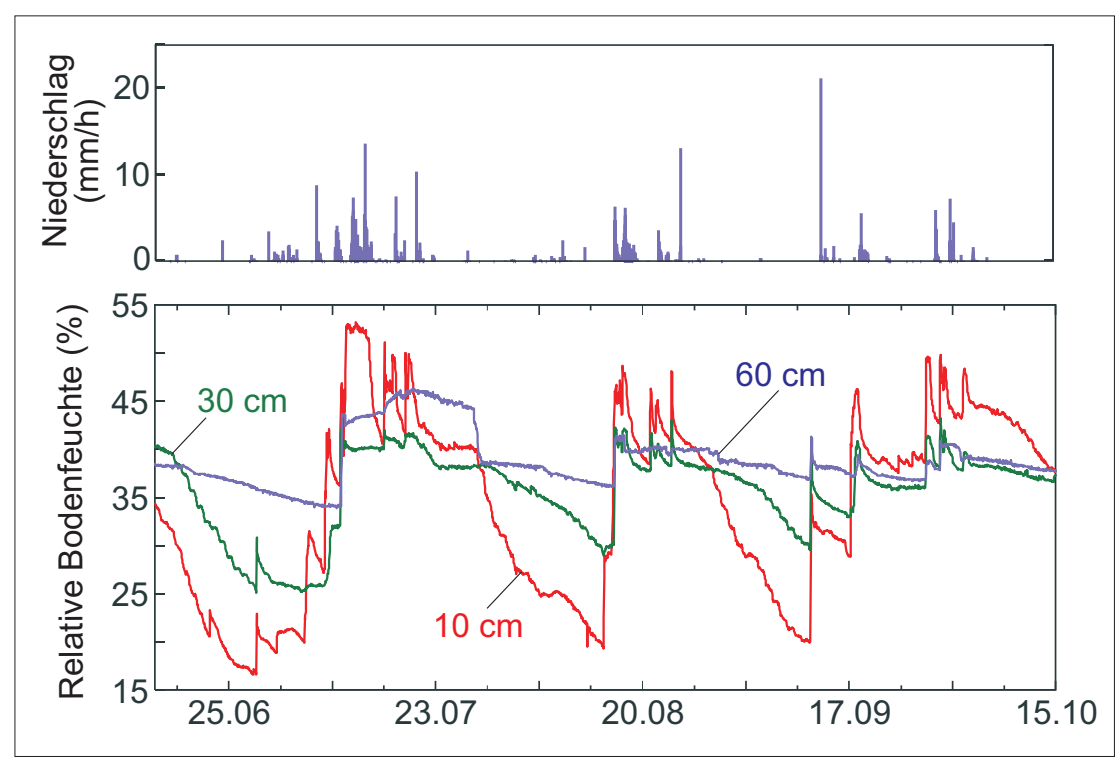

Abb. 1: Zeitlicher Verlauf der in verschiedenen Tiefen gemessenen Bodenfeuchte und des beobachteten Niederschlags an einem Wiesenstandort in Niederösterreich (Wolfsbach) für den Zeitraum von Juni bis Oktober 2006. 


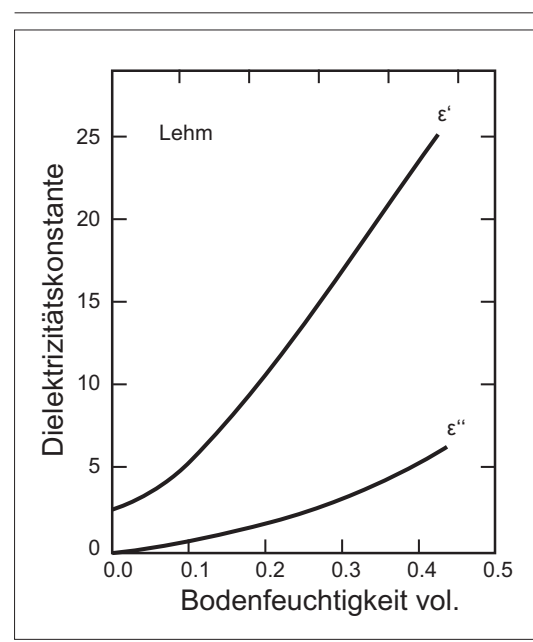

Abb. 2: Dielektrizitätskonstante einer Bodenprobe in Abhängigkeit der Bodenfeuchtigkeit bei einer Frequenz von $5 \mathrm{GHz}$, nach (Hallikainen et al. 1985).

gebnisse räumlich stark begrenzt, da es sich um Punktmessungen handelt, und die Übertragung auf Gebiete wegen der kleinräumigen Variabilität der Bodenfeuchte schwierig ist (Western et al. 2002). Im Gegensatz dazu erfolgt bei der Messung der Bodenfeuchte mit Hilfe von Fernerkundungsmethoden eine flächendeckende Erfassung des Feuchtezustandes. Die Messwerte repräsentieren hierbei jedoch nur den Feuchtezustand der oberen Bodenschicht, im Bereich von wenigen Millimetern oder Zentimetern. Auch die Häufigkeit der Messungen beschränkt sich je nach Sensor auf diskrete Zeitpunkte im Bereich von Tagen bis mehreren Wochen. Die räumliche Auflösung der gemessenen Bodenfeuchte bewegt sich je nach Sensor von $1 \mathrm{~km}$ bis $50 \mathrm{~km}$. Damit erlauben die Methoden der Fernerkundung zwar keine Aussagen über die kleinräumige Variabilität der Bodenfeuchte, enthalten jedoch Informationen über den mittleren Feuchtezustand größerer Flächen. Für hydrologische Modelle, deren räumliche Auflösung häufig größer als $1 \mathrm{~km}$ ist, stellen die gemessenen Feuchtemuster somit eine gute Ergänzung der traditionell bestehenden Daten für die Bestimmung und Validierung der Modellstruktur und der Parameter dar.

Der vorliegende Artikel gibt zunächst einen Überblick über die Methodik der Erfassung von Bodenfeuchtigkeit mittels Fernerkundung. Nach einer Vorstellung der operationellen Satellitensysteme wird auf die besonderen Anforderungen in der Hydrologie eingegangen. Anschließend werden Anwendungsbeispiele aus der praktischen Arbeit mit Satellitendaten vorgestellt.

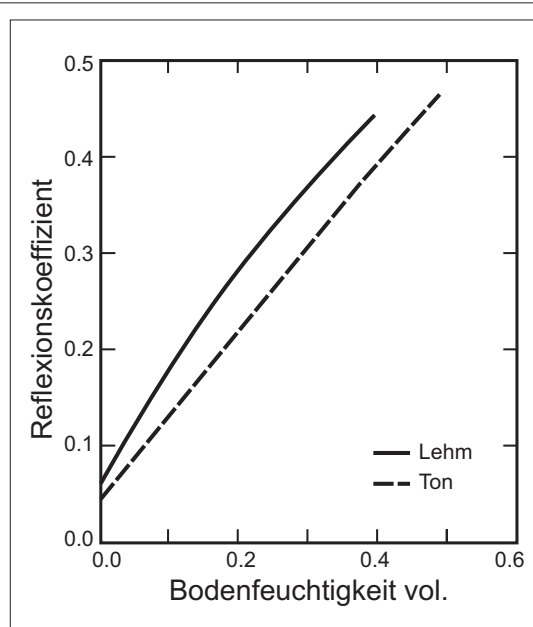

Abb. 3: Reflexionskoeffizient zweier Bodenproben in Abhängigkeit von der Bodenfeuchtigkeit bei einer Frequenz von $5 \mathrm{GHz}$, nach (Ulaby et al. 1986).

\section{Messung der Bodenfeuchte für hydrologische Anwendungen}

\subsection{Messung der Bodenfeuchte - in-situ}

Bei der in-situ Messung der Bodenfeuchte ist heute das Time-Domain-Reflectometry-Verfahren (TDR) weit verbreitet. Dabei werden Stäbe (wave guides) in den Boden eingebracht und eine Laufzeitmessung von elektromagnetischen Wellen mittels Sensoren im Bodenkörper durchgeführt. Daraus wird auf die Bodenfeuchtigkeit geschlossen. Abbildung 1 zeigt ein Beispiel für gemessene Bodenfeuchte an einer vom Hydrographischen Dienst betriebenen Messstelle in Wolfsbach/Niederösterreich. Die Messwerte zeigen deutlich die veränderliche zeitliche Dynamik der Bodenfeuchte mit zunehmender Tiefe. Während in einer Tiefe von $10 \mathrm{~cm}$ eine sehr rasche Reaktion auf große Niederschlagsintensitäten und die Verdunstung in niederschlagsfreien Zeiten ersichtlich ist, zeigen sich in 30 und $60 \mathrm{~cm}$ Tiefe deutlich gedämpfte Reaktionen.

Da die in-situ Messwerte immer nur eine Punktaufnahme im Raum darstellen und die Bodenfeuchte generell eine sehr ausgeprägte räumliche Variabilität aufweist (Western et al. 2002), ist der Rückschluss von der Punktmessung auf das weitere Umfeld der Messstelle schwierig. Um Aussagen über räumliche Muster der Bodenfeuchte treffen zu können, ist eine Verdichtung des Messnetzes notwendig. Dies ist aber mit großem Aufwand verbunden und somit nur für kleine experimentelle Testgebiete, wie z.B. das Tarrawarra-Gebiet in Australien oder das
Duero-Gebiet in Spanien, möglich (Western und Blöschl 1999; Martínez-Fernández und Ceballos 2003). Darüber hinaus gibt es weltweit nur wenige Bodenfeuchtigkeitsdatenbanken, die über konsistente und über einen längeren Zeitraum verfügbare Datensätze verfügen (Robock et al. 2000)

\subsection{Messung der Bodenfeuchte mit Methoden der Mikrowellen- fernerkundung}

\subsubsection{Physikalische Grundlagen}

Fernerkundungsgestützte Aufnahmesysteme lassen sich grundsätzlich in bodengestützte, luftgestützte und satellitengestützte Systeme unterteilen. Dabei werden die Sensoren auf Fahrzeugen oder Masten, auf Flugzeugen und Drohnen, sowie auf Satelliten angebracht. In den letzten Jahrzehnten wurden wesentliche Fortschritte in allen drei Plattformbereichen erzielt (Jackson 2005), jedoch eignen sich vor allem satellitengestützte Systeme wegen der Regelhaftigkeit der Aufnahmen für operationelle Anwendungen. Bei Mikrowellensystemen werden elektromagnetische Strahlen im Frequenzbereich von etwa 0,3 bis $30 \mathrm{GHz}$ herangezogen, was Wellenlängen von etwa $1 \mathrm{~m}$ bis $1 \mathrm{~cm}$ entspricht, die praktisch ungedämpft die Atmosphäre durchdringen können (Ulaby et al. 1981). Dies hat den entscheidenden Vorteil, dass Radarsysteme im Gegensatz zu optischen Systemen auch be Bewölkung und bei Nacht genutzt werden können.

In der Mikrowellenfernerkundung unterscheidet man nach der Art der Aufnahme zwischen aktiven und passiven Systemen. Aktive Sensoren senden elektromagnetische Pulse aus und messen die von der Erdoberfläche reflektierte Strahlung. Zu dieser Gruppe zählen etwa Radarsysteme mit synthetischer Apertur (SAR) oder Scatterometer. Im Gegensatz dazu stellt für passive Systeme (Radiometer genannt) die Energiequelle die natürliche Strahlung der Erdoberfläche selbst dar und die Sensoren sind passive Empfänger (Ulaby et al. 1982). Obwohl sich die Aufnahmetechniken unterscheiden, haben aktive und passive Systeme eine enge Verbindung durch das Kirchhoff'sche Gesetz. Dieses besagt, angewendet auf die Fernerkundung der Erdoberfläche, dass der Emissionsgrad gleich eins minus dem Reflexionsgrad ist (Schanda 1986) und somit beide Systeme im Prinzip von den selben physikalischen Phänomenen auf der Erdoberfläche betroffen sind. 
Mit zunehmendem Gehalt an Wasser im Boden steigt die Dielektrizitätskonstante an, wodurch die eintreffende Strahlung stärker gestreut bzw. zum Sensor zurück reflektiert wird. Abbildung 2 macht diesen Zusammenhang deutlich und zeigt den Real- und Imaginärteil der Dielektrizitätskonstante am Beispiel eines Bodens bei $5 \mathrm{GHz}$ aus einem Feldexperiment im US-Staat Kansas.

Unter Anwendung der Fresnel'schen Formeln lässt sich aus der Dielektrizitätskonstante der Reflexionskoeffizient berechnen, wie in Abbildung 3 dargestellt wird. Während bei trockenem Erdreich nur etwa $5 \%$ der eintreffenden Strahlungsenergie zurückgestreut und der Rest im Boden absorbiert wird, liegt der Reflexionskoeffizient bei gesättigtem Boden bei etwa $45 \%$. Aufgrund dieses relativ direkten Zusammenhangs besteht auch eine hohe Korrelation zwischen Bodenfeuchtigkeit und dem am Sensor gemessenem Rückstreukoeffizienten. Allerdings spielt eine Vielzahl an weiteren Parametern wie Einfallswinkel und Frequenz der Strahlung, sowie Vegetation und Bodenrauigkeit eine Rolle.

Die Eindringtiefe in den Boden nimmt generell mit zunehmender Frequenz und Feuchtegehalt ab und liegt laut Modellberechnungen im Bereich von wenigen Zehnteln der Wellenlänge (also im Bereich von einigen Millimetern). Des- halb eignen sich vor allem Sensoren mit Frequenzen unter $10 \mathrm{GHz}$, da damit Eindringtiefen bis etwa $10 \mathrm{~cm}$ möglich sind (Schmugge 1985). Die Ableitung von Bodenfeuchtigkeit aus Fernerkundungsdaten wird mit einer Reihe von empirischen oder theoretischen Modellen erzielt, die die Interaktion zwischen Bodenoberfläche und elektromagnetischen Wellen und den Einfluss von Vegetation beschreiben (Attema und Ulaby 1978; Oh et al. 1992; Fung 1994; Dubois et al. 1995). Im folgenden Abschnitt wird näher auf passive und aktive Mikrowellensysteme eingegangen, die von besonderer Bedeutung für die $\mathrm{Er}$ fassung der Bodenfeuchte sind. Abbildung 4 gibt einen Überblick über aktuelle sowie derzeit in Planung befindliche Satellitenmissionen.

\subsubsection{Passive Aufnahmesysteme}

Passive Systeme messen die Strahlungstemperatur und schließen dadurch indirekt auf die Emissivität und den Reflexionskoeffizienten. Allerdings müssen dabei Bodenrauigkeitseffekte berücksichtigt werden um dann in weiterer Folge mit Hilfe der Bodentextur die Bodenfeuchtigkeit abschätzen zu können. Herausforderungen bestehen allerdings beim Einfluss der Vegetation, da die Pflanzendecke das Signal überlagert, sowie bei Einflüssen durch Interferenzen mit kommerziellen
Radiofrequenzen speziell in dichter besiedelten Gebieten der Erde. Beispiele für Radiometer sind das 1978 vom US-Verteidigungsministerium gestartete Scanning Multichannel Microwave Radiometer (SMMR) mit räumlichen Auflösungen zwischen $148 \mathrm{~km}$ und $27 \mathrm{~km}$. Seit 2002 befindet sich das Advanced Microwave Scanning Radiometer (AMSR-E) der National Aeronautics and Space Administration (NASA) im All, das mit räumlichen Auflösungen von $56 \mathrm{~km}$ bzw. $38 \mathrm{~km}$ arbeitet. In Planung befindet sich derzeit die Soil Moisture Active and Passive Mission (SMAP) der NASA mit räumlichen Auflösungen von $30-40 \mathrm{~km}$, sowie die Mission Soil Moisture and Ocean Salinity (SMOS) der Europäischen Weltraumbehörde (ESA). Der Start der SMOS Mission ist für Herbst 2009 geplant und stellt die erste passive Mission im All dar, die speziell für die Erfassung der Bodenfeuchtigkeit konzipiert wurde. Die räumliche Auflösung wird je nach Einfallswinkel zwischen 30 und $50 \mathrm{~km}$ betragen und durch die hohe Überflugrate wird eine globale Abdeckung mit Bodenfeuchtedaten innerhalb von drei Tagen erzielt (Kerr et al. 2001).

\subsubsection{Aktive Aufnahmesysteme}

Zu den aktiven Systemen zählen Radarsysteme mit synthetischer Apertur (SAR) Da die geometrische Auflösung eine Funk-

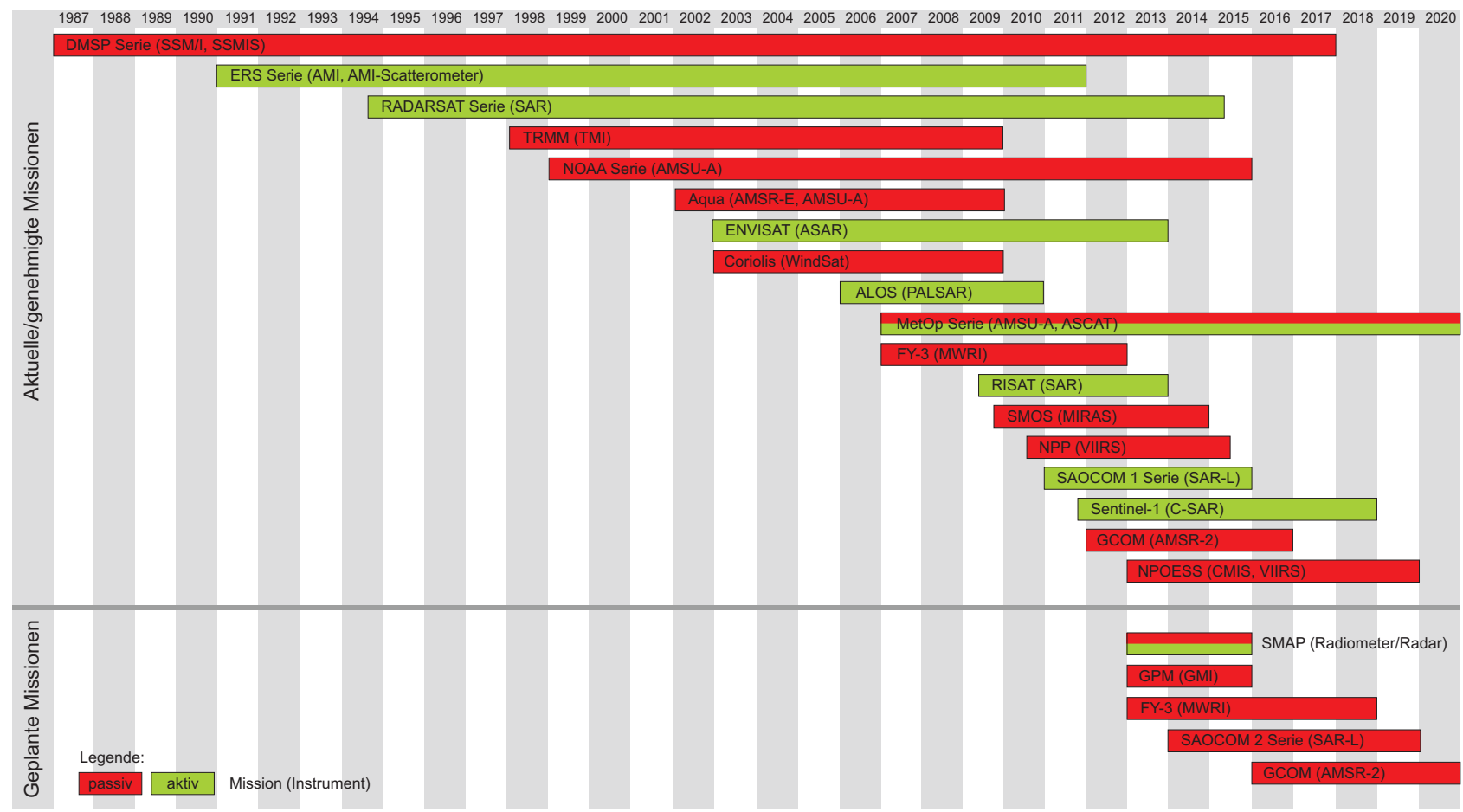

Abb. 4: Aktuelle operationelle bzw. geplante Fernerkundungsmissionen mit Relevanz für die Bodenfeuchtigkeit. Angegeben sind die Missionsnamen, sowie deren Instrumente in Klammer. Passive Systeme sind in rot, aktive Systeme in grün dargestellt. Modifiziert nach (CEOS 2008). 


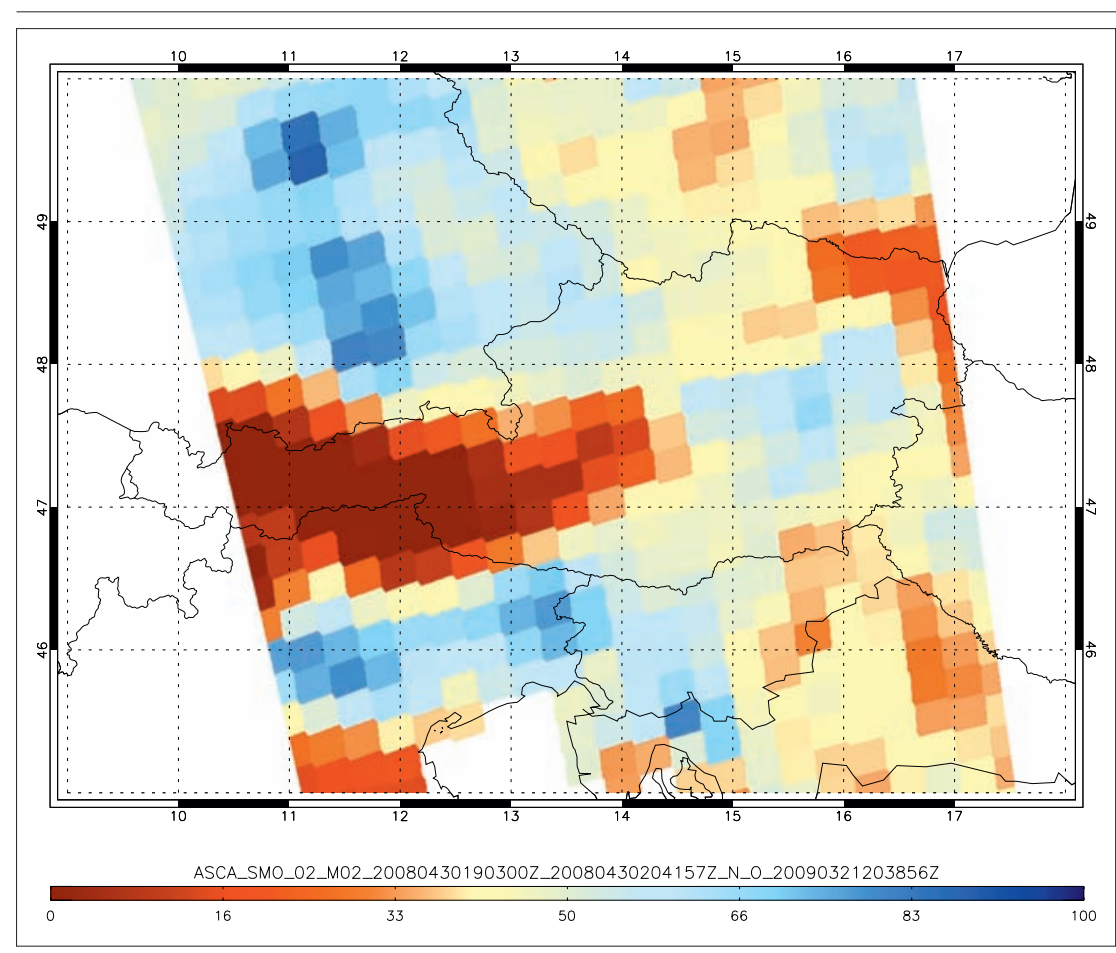

Abb. 5: Relative Bodenfeuchtigkeit (in Prozent) abgeleitet von ASCAT. Dargestellt ist der Überflug vom 30. April 2008 mit einer räumlichen Auflösung von $50 \mathrm{~km}$. Die Breite eines der beiden Aufnahmestreifen entspricht etwa $550 \mathrm{~km}$ und deckt damit nahezu ganz Österreich ab.

tion der Antennenbaulänge ist, sind Auflösungen im Bereich von wenigen Metern nur mit Antennenlängen von mehreren $\mathrm{km}$ möglich, was bei Satelliten praktisch nicht durchführbar ist. Deshalb behelfen sich SAR-Systeme dadurch, dass die Geländeoberfläche mit einer kurzen Antenne (bis etwa $10 \mathrm{~m}$ ) wiederholt bestrahlt wird und dieselben Geländepunkte somit mehrfach entlang der Flugbahn aufgenommen werden. Eine simultane Verwendung mehrerer kurzer Antennenaufnahmen ermöglicht dadurch denselben Informationsgehalt einer scheinbar langen (synthetischen) Antenne, und dadurch wird eine Erhöhung der Auflösung auf etwa $30-100 \mathrm{~m}$ erzielt. Allerdings erfordern diese Systeme einen hohen technischen Aufwand sowohl bei der Aufnahme als auch Verarbeitung der Daten und eine erhöhte Energieversorgung des Instruments, was wiederum die Aufnahmezeit und damit die räumliche Abdeckung limitiert. Des Weiteren beeinflusst Vegetation auch diese Systeme, weshalb es Methoden wie die "change-detection" gibt, bei der nicht die einzelnen physikalischen Streuungsmechanismen untersucht werden, sondern die Veränderungen des rückgestreuten Signals über die Zeit durch Verwendung von langen Zeitreihen. Unter der Annahme, dass sich Bodenrauigkeit großräumig nur geringfügig ändert, müssen lediglich die zeitlichen Effekte der Ve- getation von denen der Bodenfeuchtigkeit getrennt werden (Quesney et al. 2000). Beispiele für europäische SAR-Systeme der ESA sind das seit 1991 arbeitende Active Microwave Instrument (AMI) mit $30 \mathrm{~m}$ Auflösung, sowie das Advanced SAR (ASAR) seit 2003 mit räumlichen Auflösungen von $30 \mathrm{~m}$ bis $1 \mathrm{~km}$ (je nach Aufnahmemodus).

Als weitere Gruppe aktiver Sensoren sind schließlich Scatterometer zu erwähnen. Ursprünglich zur Erfassung von Windfeldern entwickelt, konnten die Messungen auch für Rückschlüsse auf die Bodenfeuchtigkeit verwendet werden. Die Funktionsweise ist ähnlich denen von SAR-Systemen, allerdings messen diese Systeme die Eigenschaften des zurückgestreuten Signals mit einer höheren Genauigkeit und - im Gegensatz zu Antennen mit synthetischer Apertur - unter verschiedenen Einfallswinkeln. Dadurch sind etwas geringere räumliche Auflösungen im Bereich von $25-50 \mathrm{~km}$ möglich. Der Vorteil von Scatterometern liegt in einem breiteren Aufnahmestreifen (mehrere $100 \mathrm{~km}$ ), und somit einer besseren zeitlichen Abdeckung der Erdoberfläche im Bereich von 2-6 Tagen. Auch hier werden zur Ableitung von Bodenfeuchte semi-empirimeter über Rückstreukoeffizient, Vegetation und Rauigkeit berücksichtigen (Pulliainen et al. 1998; Grippa und Woodsche Modelle herangezogen, welche Para- house 2003). Ein Beispiel für Scatterometer ist das Advanced Microwave Instrument-Scatterometer (AMI-Scat), das seit 1991 mit einer Auflösung von 50 km arbeitet. Die Europäische Organisation für die Nutzung meteorologischer Satelliten (EUMETSAT) betreibt das Advanced Scatterometer (ASCAT), das als Folgeinstrument von AMI-Scat mit ähnlichen Charakteristika konzipiert wurde. Durch Verwendung von insgesamt sechs Antennen ergibt sich eine erhöhte Anzahl der Aufnahmen von etwa einer Messung pro Tag sowie eine verbesserte räumliche Auflösung von $25 \mathrm{~km}$. Darüberhinaus liefert ASCAT Bodenfeuchtedaten operationell seit 2007, die in Echtzeit verfügbar sind.

Zur Ableitung der Bodenfeuchte aus Scatterometer-Daten wurden Verfahren wie die „change-detection“-Methode entwickelt (Wagner et al. 1999; Wagner et al. 2003), bei der zunächst lange Zeitserien (im Zeitraum von 1991-2009) des Sensors AMI-Scat an Bord des European RemoteSensing Satellite (ERS) analysiert werden. Dieser Sensor empfängt das Radarsignal mit drei Antennen, die den Rückstreukoeffizienten aus verschiedenen Einfallswinkeln messen. Dadurch ist es möglich, den Einfluss der sich zeitlich ändernden Vegetation in verschiedenen Einfallswinkelbereichen zu beobachten und somit von statischen Einflüssen zu trennen. In einem weiteren Schritt werden die trockensten und feuchtesten Zeitpunkte der gesamten Zeitserien ermittelt und in einer Datenbank gespeichert. Der letzte Verarbeitungsschritt schließlich verwendet die eigentlichen Rückstreukoeffizienten und skaliert die Werte in einer Weise, sodass das Minimum dem Wert 0 und das Maximum dem Wert 100 entspricht. Diese Methode wurde auch auf andere Sensoren wie etwa ASCAT angewandt (Bartalis et al. 2007). Abbildung 5 stellt ein Beispiel für Bodenfeuchtedaten, abgeleitet vom Sensor ASCAT, über Österreich dar.

\section{Anwendungsbeispiele von Bodenfeuchtigkeitsdaten}

Das Feld der Anwendungen von Bodenfeuchtigkeitsdaten und den abgeleiteten Produkten ist weit gestreut und reicht von der Erntevorhersage über die Abflussvorhersage bis hin zur numerischen Wettervorhersage.

Im Bereich der numerischen Wettervorhersage können Bodenfeuchtedaten zur Bestimmung der Anfangsbedingungen von Modellläufen oder für Kalibrierungszwecke genutzt werden, wo- 
bei $50 \mathrm{~km}$ Auflösung für existierende und zukünftige globale Modelle ausreichend ist. Regionale und meso-skalige Modelle benötigen jedoch zumindest $25 \mathrm{~km}$ Auflösung, und man kann annehmen, dass Modelle in naher Zukunft Satellitendaten mit höherer Auflösung verarbeiten werden können (Walker und Houser 2005).

Im Bereich der hydrologischen Modellierung werden Bodenfeuchtedaten zur Verbesserung der Anfangsbedingungen verwendet, wobei nach wie vor eine große Herausforderung darin besteht, dass die Eindringtiefe von Mikrowellen relativ gering ist, während man bei der Modellierung an der vertikalen Verteilung der Feuchte im Profil interessiert ist (Walker und Houser 2001). Mit Hilfe der RichardsGleichung ist es grundsätzlich möglich, aus der Bodenfeuchte der Oberfläche die Bodenfeuchte des Profils rückzurechnen (Entekhabi et al. 1994). Da allerdings die bodenphysikalischen Größen und Randbedingungen meist nicht in ausreichendem Maßstab bekannt sind, wurden vereinfachte Zwei-Schicht-Modelle (Jackson et al. 1981) und Mehr-Schicht-Modelle entwickelt, die besser den verfügbaren Informationen entsprechen (Koster et al. 2000). Ein solches Modell wird im Folgenden anhand eines Beispiels im nördlichen Niederösterreich erläutert.

Basierend auf der Struktur eines bestehenden hydrologischen Modells für die Hochwasservorhersage im Kamptal (Blöschl et al. 2008; Reszler et al. 2008) wurde an der TU Wien ein Zwei-SchichtModell zur Simulation der Bodenfeuchtedynamik in der obersten Bodenschicht entwickelt (Komma, in Vorbereitung). Bei der ursprünglichen Modellversion handelt es sich um ein konzeptionelles, flächendetailliertes Niederschlags-AbflussModell. Die räumliche und zeitliche Diskretisierung des Modells beträgt $1 \times 1 \mathrm{~km}$ und 15 Minuten. Die Modellparameter sind unterschiedlichen Hydrotopen (Bereiche mit ähnlichen hydrologischen Eigenschaften) zugeordnet und wurden in einem fünfstufigen Prozess unter Zuhilfenahme unterschiedlicher Informationsquellen identifiziert (Reszler et al. 2008). Da bei dieser ursprünglichen Modellversion der Bodenfeuchtehaushalt nur für die gesamte Wurzelzone erfolgt, wurde das Modell um einen zusätzlichen Bodenspeicher zur Berücksichtigung der obersten Bodenschicht erweitert. Dieser Speicher steht in Kontakt mit dem Hauptspeicher, welcher die Wurzelzone repräsentiert. Der Feuchteaustausch zwischen den Speichern erfolgt über eine lineare Beziehung

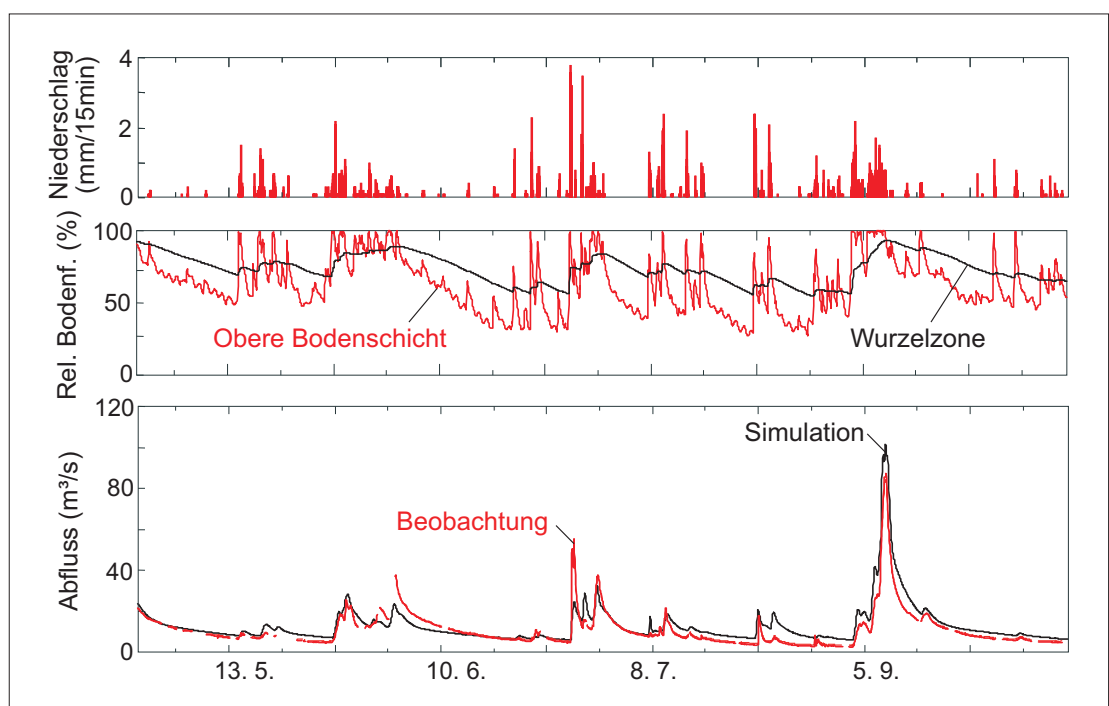

Abb. 6: Ergebnisse der hydrologischen Modellierung für das Modellgebiet Zwettl/Kamp $\left(622 \mathrm{~km}^{2}\right) \mathrm{im}$ Zeitraum Mai bis September 2006. Oben: Gebietsniederschlag. Mitte: Zeitlicher Verlauf der simulierten Bodenfeuchte für die oberste Bodenschicht und die gesamte Wurzelzone (Gebietsmittelwerte). Unten: Simulierte und beobachtete Abflussganglinie am Pegel Zwett//Kamp.

in Abhängigkeit der Feuchtedifferenz der beiden Speicher. Bei der Parametrisierung des Zweischicht-Modells wird auf die realistische Simulation der zeitlichen Bodenfeuchtedynamik in der obersten Schichte geachtet. Als Anhaltspunkt dienen hierfür die Bodenfeuchtemessungen in unterschiedlichen Tiefen von verschiedenen insitu Messstellen. Abbildung 6 zeigt die Simulationsergebnisse des adaptierten hydrologischen Modells für die Pegelmessstelle Zwettl/Kamp $\left(622 \mathrm{~km}^{2}\right)$ und den Zeitraum von Mai bis September 2006. Die Gebietsmittelwerte der simulierten Bodenfeuchte in der obersten Bodenschicht und der Wurzelzone sind im mittleren Teil der Abbildung dargestellt, der simulierte und beobachtete Abfluss im unteren Teil. Während der Feuchteverlauf in der obersten Bodenschichte eine sehr schnelle Reaktion auf den gefallenen Niederschlag aufweist, zeigt sich für die Wurzelzone eine deutlich gedämpfte Reaktion. Somit stellt sich im Modell ein ähnliches Verhalten ein, wie dies auch bei den gemessenen Werten der Bodenfeuchte (Abbildung 1) der Fall ist. Der Vergleich der simulierten Bodenfeuchte mit Scatterometer-Messungen im Zeitraum 1993-2005 zeigt grundsätzlich eine gute Übereinstimmung der räumlichen Muster von Simulation und Messung, wobei die simulierten und gemessenen Werte während der Sommermonate tendenziell besser korreliert sind.

Die Bodenfeuchtedaten lassen sich nun in konsistenter Weise in hydrologische Modelle integrieren, um die Modellannahmen zu verbessern. Dieser Vorgang wird als Datenassimilation bezeichnet. In einer Studie von (Parajka et al. 2009) wurden ERS-Scatterometerdaten in ein hydrologisches Modell auf der regionalen Skale assimiliert. Dabei wurde das Zwei-SchichtModell für 148 Einzugsgebiete in Österreich über den Zeitraum von 1991-2000 angewendet. Die Schätzung der Modellparameter erfolgte für jedes Einzugsgebiet basierend auf einem Optimierungsalgorithmus nach drei unterschiedlichen Vorgangsweisen. Im ersten Fall erfolgt die Parameterschätzung ausschließlich anhand von beobachteten Abflussmessungen; im zweiten Fall ausschließlich anhand von ERS-Scatterometerdaten; und im dritten Fall anhand einer gewichteten Zielfunktion, welche sowohl die Güte der Abflusssimulation als auch die Übereinstimmung der simulierten Bodenfeuchte mit den Scatterometerdaten berücksichtigt. Zur Beurteilung der Modelleffizienz (ME) wird das Nash-Sutcliffe-Kriterium herangezogen, wobei perfekte Modellsimulationen den Wert 1 liefern. Abbildung 7 (oben) zeigt die räumlichen Muster der erzielten Modellgüte der Abflusssimulationen, Abbildung 7 (unten) die Korrelationen von simulierter und Scatterometer-Bodenfeuchte in der oberen Bodenschicht. In den linken Abbildungsteilen wurden nur Abflussdaten für die Eichung verwendet, rechts wurden zusätzlich Fernerkundungsdaten einbezogen. Durch das Einbeziehen der Bodenfeuchtedaten ändert sich die Güte der Abflusssimulation kaum, während die Korrelation der simulierten und beobachteten Bodenfeuchte deutlich zunimmt. 


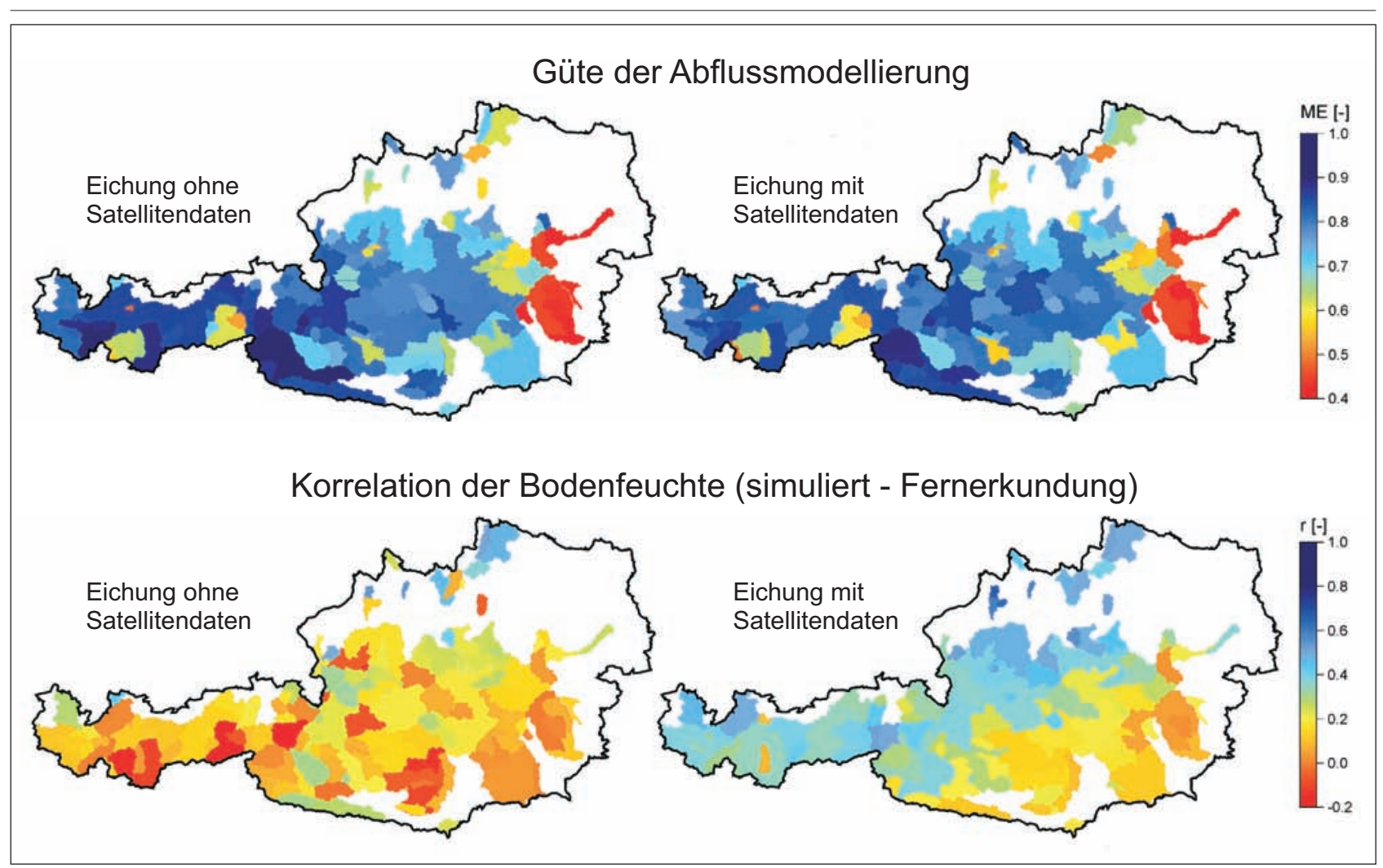

Abb. 7: Güte des Abflussmodells (oben) und der Korrelation der Bodenfeuchte zwischen dem hydrologischen Modell und Fernerkundungsdaten (unten). Der linke Teil der Abbildung zeigt jeweils die Ergebnisse für die Eichung der Modellparameter anhand von beobachteten Abflüssen, im rechten Teil werden zusätzlich Fernerkundungsdaten der Bodenfeuchte für die Eichung herangezogen.

Damit können durch die zusätzliche Einbindung der Scatterometer-Bodenfeuchte in den Eichprozess systematische Fehler bei der modellinternen Simulation der Bodenfeuchte verringert werden und realistischere Modellparameter identifiziert werden.
Die saisonale Variation der Korrelation von simulierter und ScatterometerBodenfeuchte in der obersten Bodenschicht für die drei Eichvarianten ist in Abbildung 8 dargestellt. Die Ergebnisse sind in eine Eichperiode (Abbildung 8 links) und eine Verifikationsperiode $(A b$ -

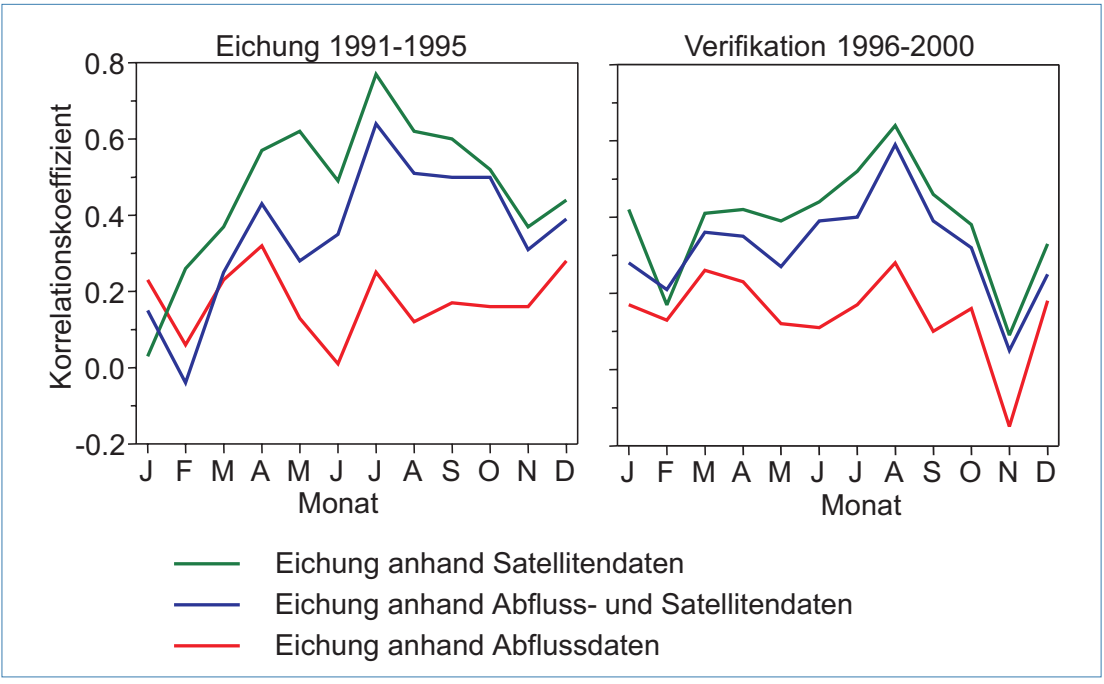

Abb. 8: Saisonale Unterschiede der Korrelation zwischen simulierter Bodenfeuchte und Scatterometer-Bodenfeuchte, dargestellt für die Eichperiode (links) und Verifikationsperiode (rechts). Die Ergebnisse basieren auf der Untersuchung von 148 Einzugsgebieten in Österreich. Für die Parametereichung wurden drei Fälle unterschieden: (1) Eichung anhand der Scatterometer-Bodenfeuchte, (2) Eichung anhand der beobachteten Abflussganglinien, und (3) Eichung unter Berücksichtigung von Abflussdaten und Scatterometer-Bodenfeuchte.

bildung 8 rechts) unterteilt. Für den Fall der Eichung ausschließlich anhand der Abflussdaten ergeben sich deutlich geringere Korrelationen der simulierten und gemessenen Bodenfeuchte. Die Tendenz zu größeren Korrelationen in den Sommermonaten ist sowohl für die Eichperiode als auch für die Verifikationsperiode ersichtlich. Insgesamt ist zu erwarten, dass ein Modell, das auf Basis eines umfangreichen Bodenfeuchtedatensatzes erstellt wurde, die Prozesse für unterschiedliche Situationen besser beschreibt als ein Modell, das ausschließlich Abflussdaten für die Eichung und den Betrieb verwendet.

\section{Zusammenfassung und Ausblick}

Die Fernerkundung bietet mehrere Möglichkeiten, die Bodenfeuchtigkeit vom Satellit aus zu beobachten. Gerade Mikrowellensensoren sind aufgrund der verwendeten Wellenlängen dazu geeignet, Informationen für die obersten Bodenschichten zu liefern. Derzeit sind Daten von Mikrowellensensoren im Skalenbereich von $25-50 \mathrm{~km}$ operationell verfügbar, welche die gesamte Erdoberfläche in wenigen Tagen vollständig abdecken. Gerade in jüngster Zeit wurden viel- 
versprechende Ergebnisse erzielt, Daten auch in einem größeren Maßstab (etwa $1 \mathrm{~km}$ ) verfügbar zu machen. Die Verfügbarkeit für operationelle Anwendungen ist bei Sensoren wie ASCAT gegeben, da Daten nicht nur in Echtzeit geliefert werden, sondern diese Mission bereits bis etwa 2020 geplant ist, und damit auch den Aufbau langer Bodenfeuchte-Zeitreihen ermöglicht.

Im Rahmen der hydrologischen Modellierung stellen Bodenfeuchtedaten eine wertvolle Zusatzinformation dar. Anhand eines Beispiels konnte gezeigt werden, dass Bodenfeuchteinformationen die Identifikation von realistischen Modellparametern erleichtern und die Gefahr von systematischen Fehleinschätzungen bei der Simulation des modellinternen Bodenfeuchtehaushalts reduzieren. Die realistische Wahl der Modellstruktur und Pa- rameter stellt eine Voraussetzung für die zutreffende Beschreibung von Extremsituationen dar, die im Regelfall nicht im Eichdatensatz enthalten sind.

Weiterer Forschungsbedarf besteht auf dem Gebiet des Downscalings von Bodenfeuchtigkeit, sowie der Verbesserung der Assimilation von Bodenfeuchtedaten im hydrologischen Modell. Im Rahmen des Projekts Satellite Application Facility on Support to Operational Hydrology and Water Management (H-SAF) wird derzeit versucht, bestehende Bodenfeuchteprodukte von Scatterometerdaten hinsichtlich ihrer Anwendbarkeit für hydrologische Aufgabenstellungen weiterzuentwickeln. Dabei werden unter anderem Downscaling-Methoden untersucht, die sich mit der Übertragung des Informationsgehalts von $25 \mathrm{~km}$ auf $1 \mathrm{~km}$ beschäftigen, um in weiterer Folge verbesserte Bodenfeuchteprodukte zu entwickeln.

\section{Danksagung}

Die Autoren bedanken sich bei der Österreichischen Akademie der Wissenschaften (Programm Hydrologie Österreichs) sowie bei den Verantwortlichen des Projekts Satellite Application Facility on Support to Operational Hydrology and Water Management, in dessen Rahmen eine Visiting Scientist Activity durchgeführt wird. Für die Bereitstellung von Daten wird dem Hydrographischen Dienst in Österreich gedankt.

\section{Korrespondenz:}

Mag. Stefan Hasenaue

Institut für Photogrammetrie und Fernerkundung TU Wien

Gußhausstrasse 27-29, 1040 Wien

Tel.: +43 15880112241
E-Mail: sh@ipf.tuwien.ac.at

\section{LITERATUR}

Attema E, Ulaby FT (1978) Vegetation modeled as water cloud. Radio Science, 13(2), 357-364. Bartalis Z, Wagner W, Naeimi V, Hasenauer S Scipal K, Bonekamp H, Figa J, Anderson C (2007) Initial soil moisture retrievals from the METOP-A Advanced Scatterometer (ASCAT). Geophysical Research Letters, 34(20). Blöschl G, Reszler C, Komma J (2008) A spaBösch G, Reszler C, Komma J (2008) A spatially distributed flash flood forecasting model. Environmental Modelling and Software, 23(4),
464-478.

CEOS (2008) Earth Observation Handbook. Retrieved 20 April 2009, from http://www.eohandbook.com/eohb2008/index.html. Dubois PC, van Zyl J, Engman (1995) Measuring soil moisture with imaging radars. Geoscience and Remote Sensing, IEEE Transactions on, 33(4), 915-926. Entekhabi D, Nakamura H, Njoku EG (1994) Solving the inverse problem for soil moisture and temperature profiles by sequential assimilation of multifrequency remotely sensed observations. IEEE Transactions on Geoscience servations. IEEE Transactions on Geoscie and Remote Sensing, 32(2), 438-448. sion models and their applications, Artech House, Boston, MA

Grippa M, Woodhouse IH (2003) Retrieval of bare soil and vegetation parameters from wind scatterometer measurements over three different climatic regions. Remote Sensing of Environment, 84(1), 16-24.

Hallikainen MT, Ulaby FT, Dobson MC, EIRayes MA, Lil-Kun W (1985) Microwave Dielectric Behavior of Wet Soil-Part 1: Empirical Models and Experimental Observations. Geoscience and Remote Sensing, IEEE Transactions on, GE-23(1), 25-34.

tions on, GE-23(1), 25-34.
Jackson TJ (2005) Estimation of surface soil moisture using microwave sensors. Encyclopedia of Hydrological Sciences, Anderson MG, John Wiley \& Sons, Chichester, 799-810. Jackson TJ, Schmugge TJ, Nicks AD, Coleman GA, Engman ET (1981) Soil moisture updating and microwave remote sensing for hydrological simulation (Oklahoma, USA).
Hydrological Sciences Bulletin, 26(3), 305-319. Kerr YH, Waldteufel P, Wigneron JP, Martinuzzi JM, Font J, Berger M (2001) Soil moisture retrieval from space: The Soil Moisture and Ocean Salinity (SMOS) mission. IEEE Transactions on Geoscience and Remote Sensing, 39(8), 1729-1735.

Koster RD, Suarez MJ, Ducharne A, Stieglitz M, Kumar P (2000) A catchment-based approach to modeling land surface processes in a general circulation model 1. Model structure. general circulation model 1. Model structure. Journal of Geophysical Research

res, 105(D20), 24809-24822. Martínez-Fernández J, Ceballos A (2003) TemExperiment in Spain. Soil Science Society of America Journal, 67(6), 1647-1656.

Oh Y, Sarabandi K, Ulaby FT (1992) An empirical model and an inversion technique for radar scattering from bare soil surfaces. Geoscience and Remote Sensing, IEEE Transactions on, 30(2), 370-381.

Parajka J, Naeimi V, Blöschl G, Komma J (2009) Matching ERS scatterometer based soil moisture patterns with simulations of a concepmoisture patterns with simulations of a concepHydrology and Earth System Sciences, 13(2), 259-271.

Pulliainen JT, Manninen T, Hallikainen MT (1998) Application of ERS-1 wind scatterometer data to soil frost and soil moisture monitoring in boreal forest zone. Geoscience and Remote Sensing, IEEE Transactions on, 36(3), 849-863. Quesney A, Le Hégarat-Mascle S, Taconet O, Vidal-Madjar D, Wigneron JP, Loumagne C, Normand M (2000) Estimation of watershed soil moisture index from ERS/SAR data. Remote moisture index from ERS/SAR data. Remote Sensing of Environment, 72(3), 290-303.
Reszler C, Komma J, Blöschl G, Gutknecht D (2008) Dominante Prozesse und Ereignistypen zur Plausibilisierung flächendetaillierter Niederschlag-Abflussmodelle. Hydrologie und Wasserbewirtschaftung, 52(3), 120-131.

Robock A, Vinnikov KY, Srinivasan G, Entin JK, Hollinger SE, Speranskaya NA, Liu S, Namkhai A (2000) The Global Soil Moisture
Data Bank. Bulletin of the American Meteorological Society, 81(6), 1281-1299.

Schanda E (1986) Physical Fundamentals of Remote Sensing, Springer, Berlin.

Schmugge TJ (1985) Remote sensing of soil moisture. Encyclopedia of Hydrological Forecasting, Anderson M, John Wiley \& Sons, Chichester, 101-124.

Ulaby FT, Moore RK, Fung AK (1981) Microwave remote sensing fundamentals and radiometry. Microwave remote sensing: active and passive, Artech House, Boston, MA, 1-456. Ulaby FT, Moore RK, Fung AK (1982) Radar remote sensing and surface scattering and emission theory. Microwave remote sensing: active and passive, Artech House, Boston, MA, 457-1064.

Ulaby FT, Moore RK, Fung AK (1986) From

theory to applications. Microwave remote sensing: active and passive, Artech House, Boston, MA, 1065-2162.

Wagner W, Lemoine G, Rott H (1999) A method for estimating soil moisture from ERS Scatterometer and soil data. Remote Sensing of Enterometer and soil data. Rem
vironment, 70(2), 191-207.

vironment, 70(2), 191-207.
Wagner W, Scipal K, Pathe C, Gerten D, Lucht W, Rudolf B (2003) Evaluation of the agreement between the first global remotely sensed soil moisture data with model and precipitation data. Journal of Geophysical Research, 108(D19). Walker JP, Houser PR (2001) A methodology for initializing soil moisture in a global climate model: Assimilation of near-surface soil moisture observations. Journal of Geophysical Research observations. Journal of Geophysical Resear

Walker JP, Houser PR (2005) Hydrologic data Walker JP, Houser PR (2005) Hydrologic data
assimilation, Chapter 2. Advances in Water Sciassimilation, Chapter 2. Adva
ence Methodologies, 25-48. ence Methodologies, 25-48. Western AW, Blöschl G (1999) On the spatial scaling of soil moisture. Journal of Hydrology, 217(3-4), 203-224.

Western AW, Grayson RB, Blöschl G (2002) Scaling of soil moisture: A hydrologic perspective. Annual Review of Earth and Planetary Sciences, 30, 149-180. 